\title{
Myriad manifestations of gallbladder carcinoma on multidetector computed tomography: Retrospective study of 230 patients from tertiary care center of North India
}

\author{
Varun Gupta1, Nishant Lohia², Harish S ${ }^{3}$, Milind B Sawant ${ }^{4}$, Yogesh Kumar Bharadwaj ${ }^{5}$ \\ ${ }^{1}$ Assistant Professor, ${ }^{4}$ Associate Professor, ${ }^{5}$ Post-graduate resident, Department of Radiology, ${ }^{3}$ Professor, Department \\ of Surgical Oncology, Command Hospital (CC), Lucknow, Uttar Pradesh, ${ }^{2}$ Associate Professor, Department of \\ Radiation Oncology, Command Hospital Air Force, Bengaluru, Karnataka, India
}

\section{A B S T R A C T}

Background: Gallbladder carcinoma is endemic in India. Though imaging findings of gallbladder carcinoma are very well-described in the literature, the research question that guided this study was to evaluate various imaging manifestations of the disease in this particular geographic region of North India which is a highly endemic region for gallbladder cancer and compare with studies from other parts of the world. Aims and Objectives: The purpose of the study was to retrospectively analyze the spectrum of findings on Multidetector Computed Tomography (MDCT) in histologically proven gallbladder carcinoma detected at a tertiary care center from the NorthIndia. The primary objective was to define the various patterns of growth while the secondary objective was to analyze the specific prevalence and patterns of spread of the disease. Materials and Methods: We retrospectively studied Contrast-Enhanced Computed Tomography findings in 230 patients of histologically proven gallbladder carcinoma. Patients with previous cholecystectomy or biliary intervention were excluded from the study. Results: In our study, focal or asymmetrical wall thickening of the gallbladder was the most common growth pattern seen in 140 (61\%) patients. Contiguous infiltration of the liver was seen in $190(83 \%)$ cases. Regional nodal involvement was observed in 90 (39\%) patients, while 100 (43\%) patients had both regional and distant nodal involvement. Liver and peritoneal metastases were noted in $71(31 \%)$ patients and 96 (42\%) patients, respectively. Majority (81\%) of patients had Stage IV disease. Conclusion: MDCT provides comprehensive information regarding the local extent as well as distant spread of gallbladder carcinoma. Asymmetric gall bladder wall thickening in this geographical region must be considered suspicious and should evoke histopathological analysis to exclude malignancy.

Key words: Gallbladder carcinoma; Metastases; Multidetector computed tomography
Access this article online

Website:

http://nepjol.info/index.php/AJMS

DOI: $10.3126 /$ ajms.v13i3.41104

E-ISSN: 2091-0576

P-ISSN: 2467-9100

Copyright (c) 2022 Asian Journal of Medical Sciences

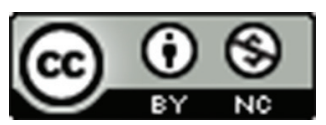

This work is licensed under a Creative Commons Attribution-NonCommercial 4.0 International License.

\section{INTRODUCTION}

Gallbladder carcinoma is the most common malignancy of the biliary system and the fifth most common malignancy of the gastrointestinal tract worldwide. ${ }^{1}$ It is an aggressive malignancy with an overall poor prognosis. Due to the non-specific nature of symptoms, most of the patients present with advanced disease. Gallbladder carcinoma is endemic in India and contributes to about $10 \%$ of global burden of gallbladder carcinoma. ${ }^{2}$ Even in India, there is significant geographic variability. North, East, North-east and Central India have higher incidence as compared to 
the South and West India. ${ }^{2}$ The common risk factors for gallbladder carcinoma include female gender, gall stones, chronic cholecystitis, porcelain gallbladder, gallbladder polyps $(>1 \mathrm{~cm})$, choledochal cyst, anomalous pancreaticobiliary junction, and chronic infections (Salmonella typhi, and liver flukes), obesity, environmental exposure to carcinogens, and genetic alterations. ${ }^{3}$

Though ultrasonography is usually the initial modality in the evaluation of the gallbladder carcinoma, it cannot be relied upon for depiction of the full extent of the disease spread. Contrast-Enhanced Computed Tomography (CECT) provides comprehensive information about the local extent of the tumor, regional and distant lymphadenopathy, and distant organ metastases. Magnetic Resonance Cholangiopancreatography is an excellent modality for depiction of biliary ductal anatomy and abnormality which helps in the pre-procedure planning of endoscopic or percutaneous transhepatic biliary drainage.

\section{Aims and objectives}

The purpose of the study was to retrospectively analyze the spectrum of findings on Multidetector Computed Tomography (MDCT) in gallbladder carcinoma detected at a tertiary care center from North India. The author's institute is located in a geographically endemic area for carcinoma gall bladder. The research question which acted as guiding force towards this study was to define the various patterns of disease growth, regional and distant nodal involvement, and metastases in this geographical region and to compare those patterns with the studies in other parts of the World, already mentioned in literature.

With this research question, the primary objective was to define the various patterns of growth of gall bladder cancer. The secondary objective was to see the specific prevalence and patterns of spread in relation to local spread, locoregional spread, and distant metastatses.

\section{MATERIALS AND METHODS}

A total number of 432 patients of suspected gallbladder carcinoma were reported to the Malignant Disease Treatment Centre of our hospital during the period from January 2016 to June 2021. Out of these 432 patients, 342 patients underwent CECT abdomen in our center. Patients with previous cholecystectomy or biliary intervention were excluded from the study population. Among these 342 patients, 230 patients had histologically proven gallbladder carcinoma by image-guided Fine Needle Aspiration Cytology/core needle biopsy/post-operative histopathology. A retrospective observational study of CECT findings was undertaken to analyze the primary and secondary objectives of this study for these 230 histopathologically proven gallbladder carcinoma patients after obtaining the approval of the institutional ethical committee.

Computed tomographic examination was performed using a 16-slice MDCT scanner (Brilliance16, Philips Healthcare). Pre-contrast scan was performed for the upper abdomen after asking the patient to drink $750 \mathrm{ml}$ of $5 \%$ iodinated oral contrast over 45-60 min and $250 \mathrm{ml}$ of oral contrast was administered on the gantry table just before the study to distend the stomach and duodenum adequately. In some patients, oral water instead of diluted iodinated contrast was used to distend the stomach and duodenum. CECT scan of abdomen and pelvis was performed during portal venous phase after the intravenous injection of $1.5 \mathrm{ml} / \mathrm{kg}$ body weight non-ionic iodinated contrast - Iohexol $350 \mathrm{mg}$ Iodine/ml (Omnipaque 350; GE Healthcare) with the pressure injector at the rate of $3.5 \mathrm{ml} / \mathrm{s}$ and post-injection scan delay of 50-60 s. The scanning parameters were as follows: $120 \mathrm{kVp}$; tube current of 150-200 effective mAs; gantry rotation time of $0.5 \mathrm{~s}$; pitch1; detector collimation of $16 \times 0.75 \mathrm{~mm}$ and field of view of $300-350 \mathrm{~mm}$. Multiplanar reconstruction was done with $2 \mathrm{~mm}$ slice thickness and $1 \mathrm{~mm}$ increment. In some cases, triplephase study was done using bolus trigger technique using the upper abdominal aorta as the region of interest and threshold density of $100 \mathrm{HU}$ for arterial phase, followed by portal venous phase at 50-60 s and venous phase at $90 \mathrm{~s}$ following contrast injection. In some cases, the chest was also included in the portal phase along with the whole abdomen.

\section{Imaging analysis}

Two radiologists having 13 years and 5 years of experience, respectively, reviewed the images in consensus. Both reviewers knew all 230 patients had confirmed gallbladder carcinoma. The following parameters were noted - (a) Pattern of growth - focal or diffuse asymmetrical wall thickening, intraluminal polypoidal growth and mass replacing gallbladder; (b) the part of gallbladder involved-fundus, body and neck; (c) Adjacent organ involvement- either obvious direct invasion or loss of intervening fat plane with liver, antro-duodenum, pancreas, hepatic flexure/proximal transverse colon; (d) Involvement of common hepatic/common bile duct with resultant upstream biliary dilatation; (e) vascular involvement - direct invasion of the portal vein or hepatic artery with resultant irregularity of the wall outline, narrowing or when tumor/lymph node mass was seen on either side of the vessel causing its encasement. (f) Lymph node involvement - regional (cystic, pericholedochal, peripancreatic, gastrohepatic, coeliac and superior mesenteric artery) nodes; distant (paraaortic/aortocaval 
or more distant) nodes. These nodes were considered positive when either size (mean short-axis diameter more than $1 \mathrm{~cm}$ ) or morphology (internal necrosis or irregular spiculated outline due to pericapsular extension) criteria were fulfilled; $(\mathrm{g})$ hepatic metastases - round-to-oval lesions appearing hypodense on the non-contrast scan and showing peripheral enhancement on portal phase; (h) Omental involvement - Omental stranding, enhancing nodularity and thickening or omental caking; (i) ascites; (j) metastases to the other organs - such as lungs, adrenals, bones, ovaries (Krukenberg tumor); (k) gall stones. Staging of the gallbladder carcinoma was done according to the $8^{\text {th }}$ edition of American Joint Committee on Cancer staging manual. ${ }^{4}$ Accordingly, the nodal station was characterized as N1 if 1-3 regional nodes were involved and N2 if 4 or more regional nodes were involved. The para-aortic, aortocaval, or more distant nodal involvement was considered as distant metastasis (M1).

\section{RESULTS}

Out of 230 patients studied, 158 (69\%) were women and $72(31 \%)$ were men. The most common age group was $61-70$ years with a median age of 61.5 years. The youngest patient was 25-year-old lady while the oldest was 90-year-old lady. The most common growth pattern was focal or asymmetric wall thickening (Figure 1) seen in 140 (61\%) patients followed by mass replacing the gallbladder (Figure $2 \mathrm{a}$ ) noted in $75(32 \%)$ patients. The intraluminal polypoidal growth (Figure 3) was observed in 15 (7\%) cases. Liver was the commonest site involved by contiguous infiltration (Figure $2 \mathrm{~b}$ ). The pancreatic involvement (Figure 4) was seen in $14(6 \%)$ cases. Seventy $(30 \%)$ patients had vascular involvement (Figure 5) at presentation. Lymphatic spread to regional nodes was observed in $90(39 \%)$ patients, while $100(43 \%)$ patients had both regional and distant nodal involvement (Figure 6a and 6b). Two patients had para-

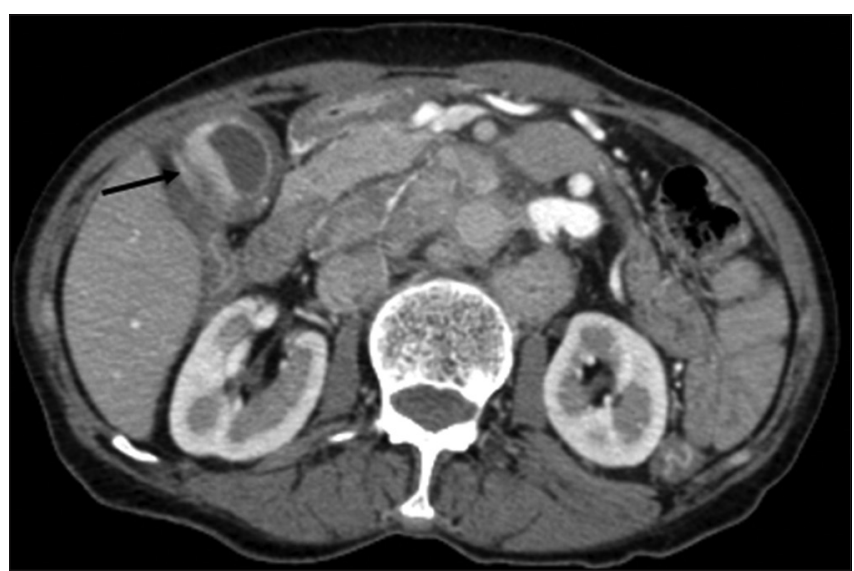

Figure 1: Asymmetric enhancing wall thickening of the gallbladder aortic lymphadenopathy without regional lymph node involvement.
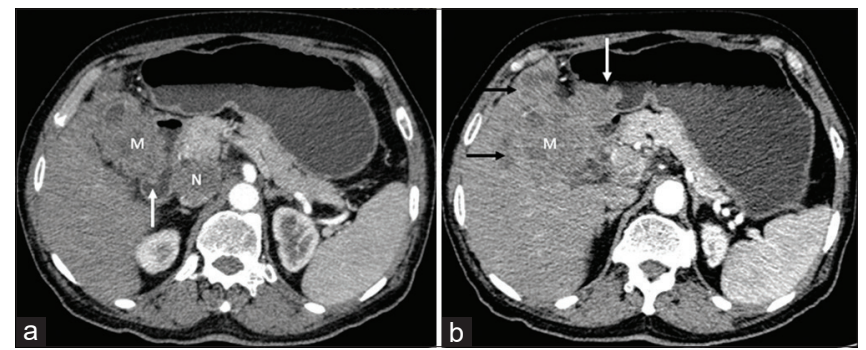

Figure 2: Mass replacing gallbladder and adjacent organ invasion: (a) axial section shows a mass $(\mathrm{M})$ replacing the gallbladder with invasion of the duodenum (white arrow). Also note pericholedochal node $(\mathrm{N})$. (b) axial section at more cranial level of the same patient shows the invasion of the adjacent liver parenchyma (black arrows) and gastric antrum (white arrow) by the GB mass

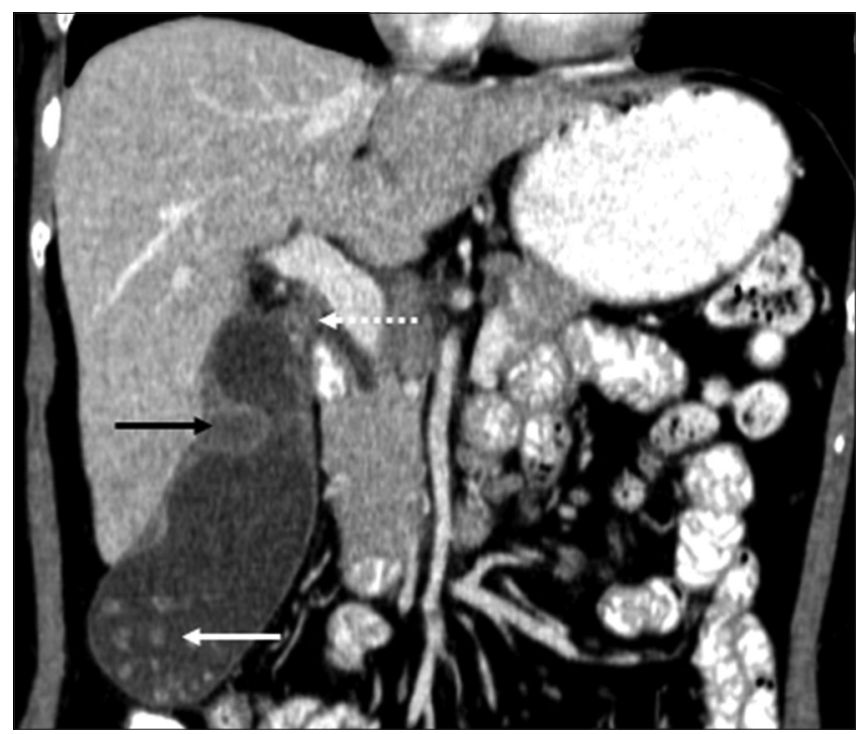

Figure 3: Coronal image showing focal polypoidal growth (black arrow), cholelithiasis (white arrow), and enhancing lesion (dashed arrow) at the neck of the gallbladder causing over distension of the gallbladder

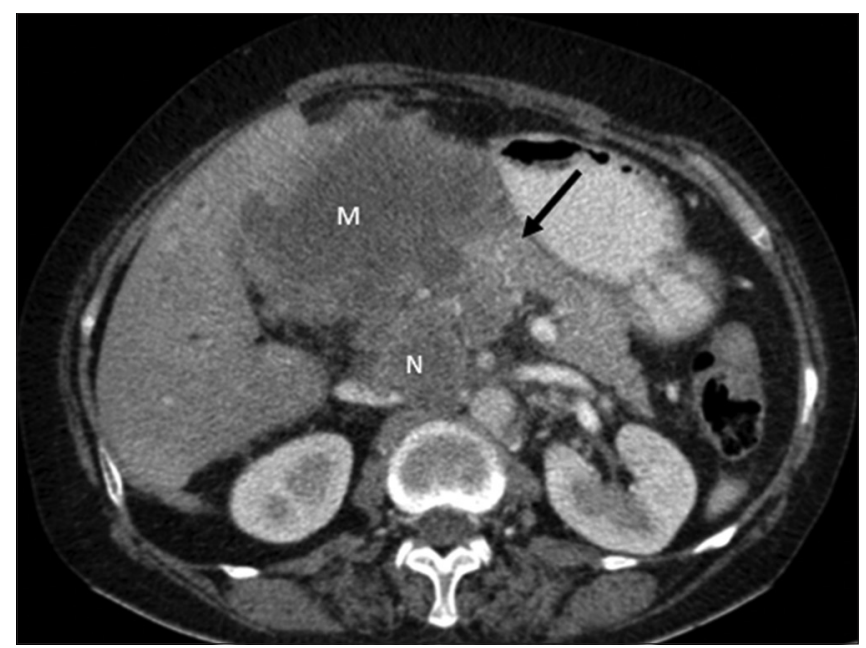

Figure 4: There is a large mass (M) arising from the gallbladder invading the pancreas (black arrow). There is also aortocaval lymph node $(\mathrm{N})$ 


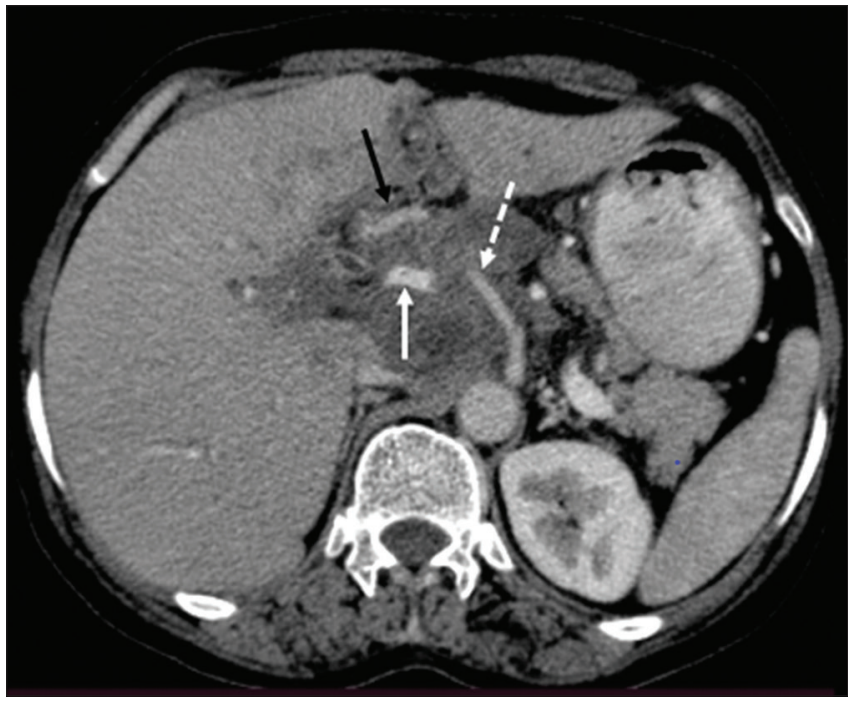

Figure 5: Vascular involvement: There is a conglomerate nodal mass at the hilum encasing portal vein (white arrow), common hepatic artery (dashed arrow), and right hepatic artery (black arrow)
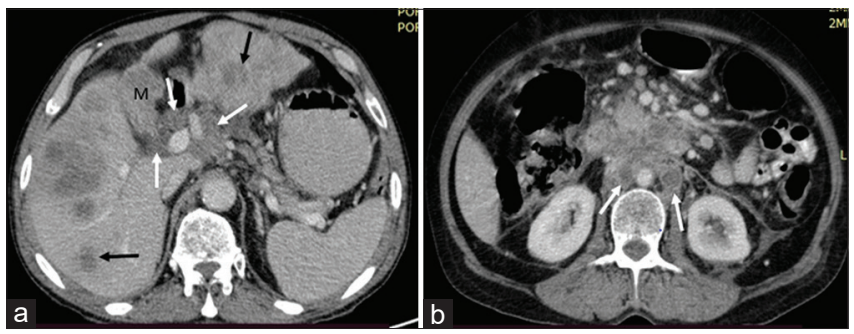

Figure 6: Nodal and hepatic metastases: (a) shows mass (M) in the gallbladder with multiple regional lymph nodes (white arrow) and hepatic metastases. (b) in the same patient shows necrotic distant nodes (white arrows)

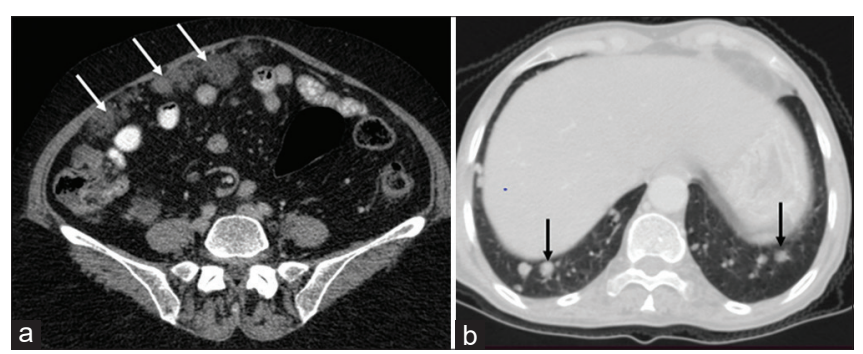

Figure 7: (a) shows multiple enhancing nodular omental deposits (white arrows). (b) shows multiple pulmonary metastases (black arrows)
Liver (Figure 6a) and omentum or peritoneum (Figure 7a) followed by lung (Figure 7b) were common sites of metastases. Ascites was noted in $70(30 \%)$ patients. Uncommon and rare sites of distant metastases (Figure 8, 9 and 10) included ovaries $(n=10)$, and adrenals $(n=5)$, spleen $(\mathrm{n}=1)$, umbilicus $(\mathrm{n}=1)$, mediastinal nodes $(\mathrm{n}=9)$, inguinal nodes $(\mathrm{n}=2)$, internal mammary node $(\mathrm{n}=1)$, supraclavicular node $(\mathrm{n}=1)$ and bones $(\mathrm{n}=5)$. One patient had mediastinal lymphadenopathy along with lung metastases without abdominal lymphadenopathy. One patient had pleural and pericardial metastases (Figure 11) along with extensive hepatic and lung metastases and mediastinal adenopathy. Skeletal metastases were noted in five $(2 \%)$ patients, out of which three had lytic, one had blastic and one had both lytic and blastic metastases. Isolated lung metastases were seen in six patients. Majority $(81 \%)$ of patients had stage IV disease. The major findings are summarised in Table 1.

\section{DISCUSSION}

Most of the literature describes mass replacing the gallbladder as the most common presentation seen in 40$65 \%$ of cases followed by focal or diffuse gallbladder wall thickening in 20-30\%, and an intraluminal polypoid mass in $15-25 \%$ cases. $^{5-10}$ In our study, focal or asymmetrical diffuse wall thickening was the most common growth pattern seen in $61 \%$ of cases. Associated gall stones were seen in $58(25 \%)$ cases, though CT is not the modality of choice as the majority of the gallbladder calculi are radiolucent and we did not take cognizance of ultrasound or MRI findings for gall stones.

Gallbladder carcinoma spreads by various modes which include direct invasion, lymphatic, hematogenous, intraperitoneal, and intraductal metastasis. Contiguous liver infiltration is a common finding in gallbladder carcinoma. The lack of submucosa in the gallbladder wall and its direct venous drainage through the liver parenchyma to the hepatic veins explains the propensity to invade the liver early in the course of the disease. ${ }^{11,12}$ Sons et al., in their autopsy

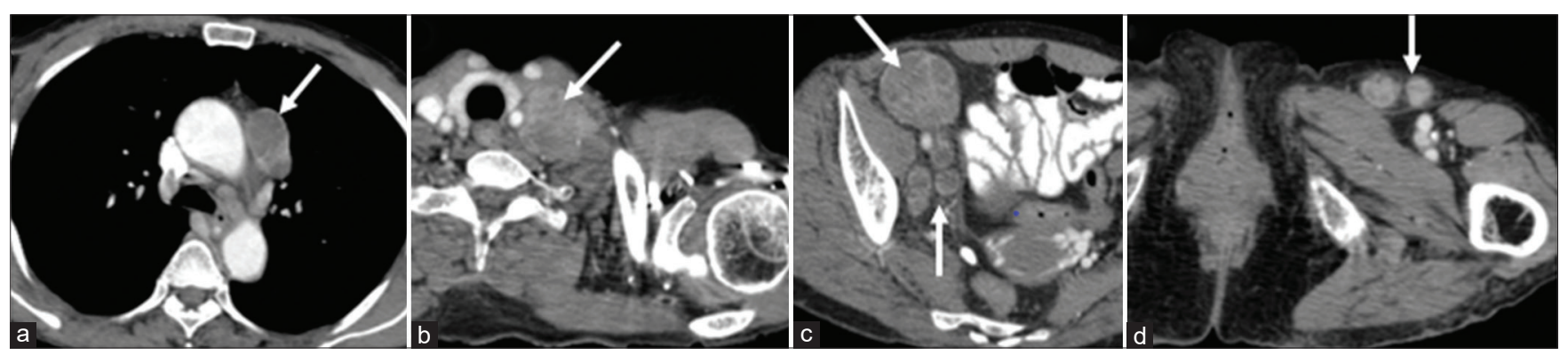

Figure 8: Uncommon distant nodal metastases (white arrows) of gallbladder carcinoma at mediastinal node in (a), supraclavicular node in (b), external and internal iliac nodes in (c), and inguinal nodes in (d) 
series of 287 patients demonstrated direct extension to the liver in $65 \%$ of cases. ${ }^{13}$ In our study, hepatic infiltration was seen in $82 \%$ of cases. This may be overassessment as loss of intervening fat plane does not always indicate invasion. The loss of fat plane can also happen due to pericholecystic adhesions due to inflammation or partial volume-averaging. Increased prevalence of liver invasion in our study may also reflect geographic variation as the disease is more common and more aggressive in North and North-East India. ${ }^{2}$ Singh et al., in their prospective study of 60 patients of gallbladder carcinoma from North India also found hepatic infiltration in $75.5 \%$ of cases. ${ }^{14}$ Gastric antrum-duodenal, colonic and pancreatic involvement was observed in $48 \%, 28 \%$, and $6 \%$, respectively, in our study. In an autopsy series, Sons et al., found antro-duodenal and colonic involvement in $15 \%$ each and pancreatic involvement in $6 \%$ of cases. ${ }^{13}$ High prevalence of adjacent organ involvement and
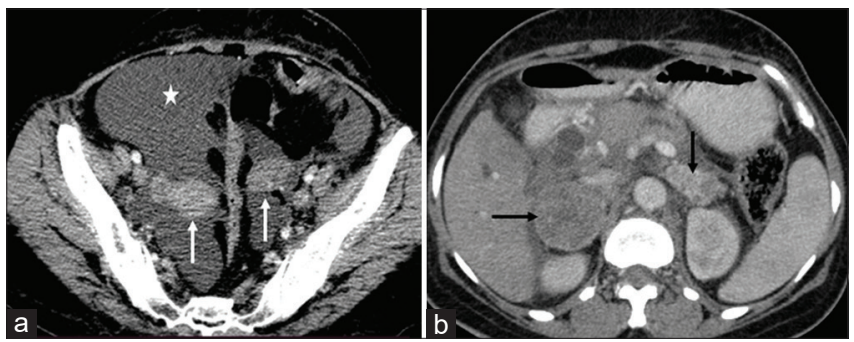

Figure 9: Uncommon metastatic sites in gallbladder carcinoma. (a) axial section at pelvic level shows enhancing deposits (arrows) in both ovaries and ascites (asterix). (b) shows heterogeneously enhancing bilateral adrenal masses in a known case of metastatic gallbladder cancer vascular invasion in our series may also be due to delayed presentation. Vascular invasion or involvement of two or more extra-hepatic organs or structures (T4 disease) precludes curative-intent surgery. The important point to be noted while considering "T-staging" is that imaging is not reliable to differentiate T1(tumor invading lamina propria or muscle layer) from T2 (tumor invading perimuscular connective tissue, but not extended beyond the serosa or into the liver) stage. ${ }^{15}$

Lymphatic spread of gallbladder cancer is common and occurs in a predictive manner. The cystic or the pericholedochal lymph nodes are the first to get involved, followed by the involvement of the celiac, superior mesenteric, and paraaortic lymph nodes. ${ }^{16}$ Surprisingly, two of our patients had paraaortic lymphadenopathy without any regional lymph node involvement. The involvement of four or more regional nodes (N2) or distant nodes (M1) precludes curative surgery. ${ }^{17}$ Ohtani et al., in their retrospective study of 59 patients reported the sensitivity of CT scan for detecting abnormal N1 and N2 nodes as $36 \%$ and $47 \%$ respectively. ${ }^{18}$ In the same study, sensitivity of CT scan for hepatic involvement more than $2 \mathrm{~cm}$ was $100 \%$ and for hepatic involvement $<2 \mathrm{~cm}$ was $65 \% .{ }^{18}$

The liver and peritoneum are common sites of distant metastasis. Kuszyk et al., reported an overall sensitivity of $91 \%$ for portal venous phase contrast-enhanced helical $\mathrm{CT}$ in detecting malignant liver tumors $>1 \mathrm{~cm}$ based on comparison with intraoperative and pathologic findings. ${ }^{19}$
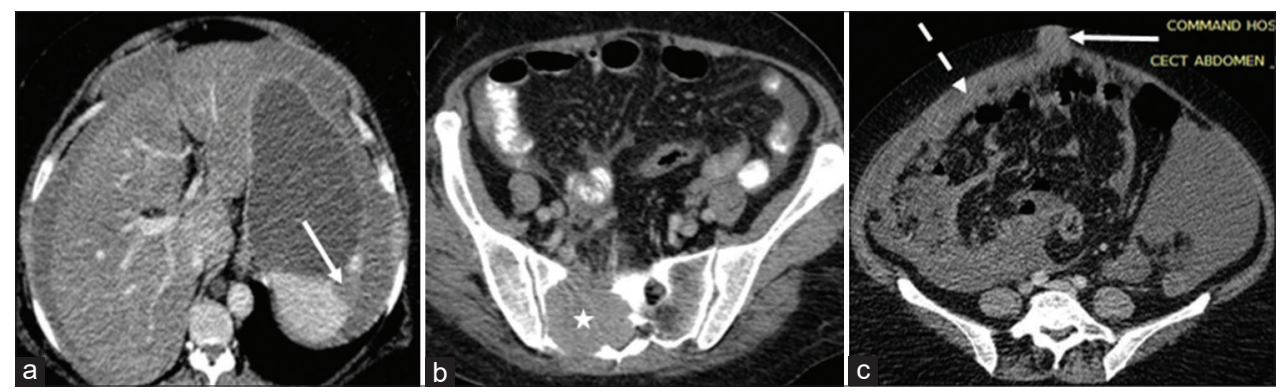

Figure 10: Rare sites of metastases in gallbladder carcinoma: CECT axial images show splenic metastasis (white arrow) in (a), sacral metastasis (asterix) in (b) and umbilical metastasis (white arrow) in (c). Also, note omental caking (white dashed arrow) along the anterior abdominal wall in (c)
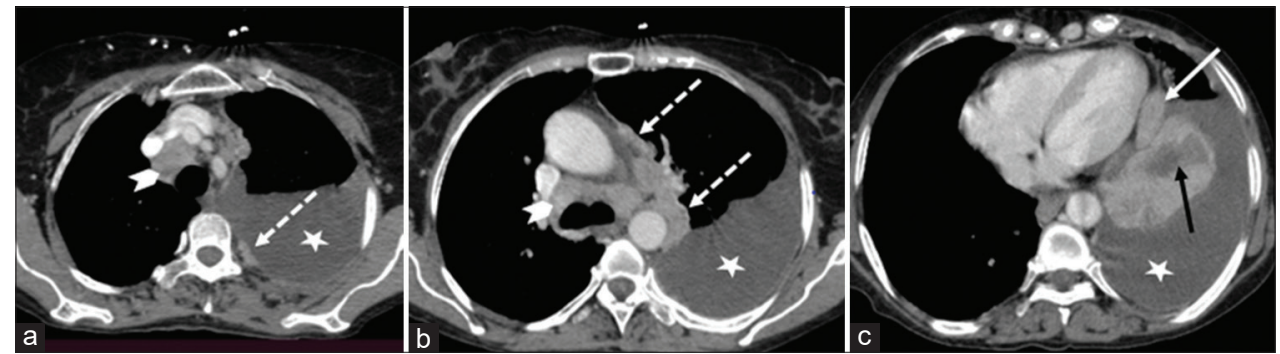

Figure 11: Pleural and pericardial metastases: CECT chest axial images in a 60-year-old lady with known gallbladder carcinoma show left pleural effusion (asterix), enhancing nodular and plaque-like pleural thickening (dashed arrows) and mediastinal nodes (arrowheads) in (a and b). (c) shows enhancing pericardial deposit (white arrow) and pulmonary metastasis (black arrow) within a collapsed left lung 


\begin{tabular}{|c|c|c|c|}
\hline Study Parameters & Range/Characteristics & No of patients $(n)$ & Percentage (\%) \\
\hline \multirow[t]{5}{*}{ Age in Years } & $\leq 40$ & 9 & $4 \%$ \\
\hline & $41-\leq 50$ & 34 & $15 \%$ \\
\hline & $51-\leq 60$ & 66 & $29 \%$ \\
\hline & $61-\leq 70$ & 75 & $32 \%$ \\
\hline & $>70$ & 46 & $20 \%$ \\
\hline \multirow[t]{3}{*}{ Growth Pattern } & Focal/Diffuse asymmetric wall thickening & 140 & $61 \%$ \\
\hline & Intraluminal polypoidal growth & 15 & $7 \%$ \\
\hline & Mass replacing GB* & 75 & $32 \%$ \\
\hline \multirow[t]{2}{*}{ Gall stones } & Present & 58 & $25 \%$ \\
\hline & Absent & 172 & $75 \%$ \\
\hline \multirow[t]{5}{*}{ Adjacent organ or structure involvement } & Infiltration of $\mathrm{CHD} / \mathrm{CBD}+$ & 132 & $57 \%$ \\
\hline & Contiguous Liver Involvement & 190 & $83 \%$ \\
\hline & Antro-duodenal Involvement & 110 & $48 \%$ \\
\hline & Colon Involvement & 64 & $28 \%$ \\
\hline & Vascular Invasion & 70 & $30 \%$ \\
\hline \multirow[t]{3}{*}{ Nodal Involvement } & No nodes involved & 40 & $18 \%$ \\
\hline & Regional Nodes & 90 & $39 \%$ \\
\hline & Regional and Distant Nodes & 100 & $43 \%$ \\
\hline \multirow[t]{3}{*}{ Metastases } & Liver Metastases & 71 & $31 \%$ \\
\hline & Omental Metastases & 96 & $42 \%$ \\
\hline & Lung Metastases & 39 & $17 \%$ \\
\hline \multirow[t]{5}{*}{ Stage } & $\|$ & 6 & $3 \%$ \\
\hline & IIIA & 21 & $9 \%$ \\
\hline & IIIB & 16 & $7 \%$ \\
\hline & IVA & 28 & $12 \%$ \\
\hline & IVB & 159 & $69 \%$ \\
\hline
\end{tabular}

*GB - Gall Bladder; +CHD/CBD - Common Hepatic Duct/Common Bile Duct

Peritoneal metastases are usually due to direct spread or "drop metastasis." MDCT has sensitivity of $85-93 \%$ for the detection of peritoneal metastases. ${ }^{20}$

In our study, we also noted some extremely rare sites of metastasis, such as the bone, spleen, pleura, pericardium, and umbilicus (Sister Mary Joseph's nodule). All these patients had extensive disease with concurrent metastases in multiple organs.

MDCT scan with its multiplanar capability is a reliable modality to stage the disease and pre-operatively assess the resectability. Kalra et al., in their study of twenty patients, reported an accuracy of $85 \%$ in predicting the resectability of gallbladder carcinoma using dual-phase MDCT. ${ }^{16}$ Sixtynine percent of our patients were found to have stage IVB reflecting the fatal nature of the disease and need for reliable screening test to detect it at an early stage.

The biggest advantage of this study is a relatively large sample size. To the best of our knowledge, no other study evaluating MDCT in gallbladder carcinoma has such a large study population.

\section{Limitations of study}

The patients included in the study were already proven cases of the carcinoma gall bladder, having varied presentations. Since the histopathological confirmation was already there, hence, the patterns of growth had to be depicted retrospectively. Moreover, the pattern of growth, contiguous organ spread, and the lymphadenopathy are best discernible on portal venous phase images. As far as the differences in scanning protocol are concerned, all patients underwent the portal venous phase while many underwent the arterial phase as well. Therefore, the differences in scanning techniques do not completely obviate the analysis. However, the authors agree that triple-phase scanning including the arterial phase is more beneficial for holistic assessment of the disease.

\section{CONCLUSION}

Gallbladder carcinoma is a dreaded malignancy with an overall abysmal prognosis. Most of the patients present with advanced disease when curative surgery is not possible. MDCT provides reliable information to stage the disease and to plan further curative or palliative management. Focal or asymmetric wall thickening in the gall bladder should always raise suspicion and further assessment to exclude neoplasm must be carried out as this was the chief pattern of growth seen in the study.

\section{REFERENCES}

1. Siegel RL, Miller KD, Fuchs HE and Jemal A. Cancer Statistics, 2021. CA Cancer J Clin. 2021;71(1):7-33.

https://doi.org/10.3322/caac.21654 
2. Dutta U, Bush N, Kalsi D, Popli P and Kapoor VK. Epidemiology of gallbladder cancer in India. Chin Clin Oncol. 2019;8(4):33 https://doi.org/10.21037/cco.2019.08.03

3. Wernberg JA and Lucarelli DD. Gallbladder cancer. Surg Clin North Am. 2014;94(2):343-360.

https://doi.org/10.1016/j.suc.2014.01.009

4. Amin MB, Greene FL, Edge SB, Compton CC, Gershenwald JE, Brookland RK, et al. The eighth edition AJCC cancer staging manual: Continuing to build a bridge from a population-based to a more "personalized" approach to cancer staging. CA Cancer J Clin. 2017;67(2):93-99.

https://doi.org/10.3322/caac.21388

5. Franquet T, Montes M, de Azua YR, Jimenez FJ and Cozcolluela R. Primary gallbladder carcinoma: Imaging findings in 50 patients with pathologic correlation. Gastrointest Radiol. 1991;16(2):143-148. https://doi.org/10.1007/bf01887330

6. Lane J, Buck JL and Zeman RK. Primary carcinoma of the gallbladder: A pictorial essay. Radiographics. 1989;9(2):209-228. https://doi.org/10.1148/radiographics.9.2.2648501

7. Yeh HC. Ultrasonography and computed tomography of carcinoma of the gallbladder. Radiology. 1979;133(1):167-173. https://doi.org/10.1148/133.1.167

8. Yum HY and Fink AH. Sonographic findings in primary carcinoma of the gallbladder. Radiology. 1980;134(3):693-696 https://doi.org/10.1148/radiology.134.3.7355220

9. Levy AD, Murakata LA and Rohrmann CA Jr. Gallbladder carcinoma: Radiologic-pathologic correlation. Radiographics. 2001;21(2):295-314; questionnaire, 549-55. https://doi.org/10.1148/radiographics.21.2.g01mr16295

10. Gul P, Gul P, Altaf $O$, Kaimkhani M and Asghar H. Spectrum of computed tomography findings in gall bladder carcinoma patients: A retrospective observational study from tertiary care oncology setup. Int Surg J. 2020;7(7):2091-6.

http://doi.org/10.18203 / 2349-2902.isj20202809

11. Kanthan R, Senger JL, Ahmed S and Kanthan SC. Gallbladder cancer in the $21^{\text {st }}$ Century. J Oncol. 2015;2015:967472.

https://doi.org/10.1155/2015/967472

12. Hussain HM, Little MD and Wei S. AIRP best cases in radiologic- pathologic correlation: Gallbladder carcinoma with direct invasion of the liver. Radiographics. 2013;33(1):103-108. https://doi.org/10.1148/rg.331115163

13. Sons HU, Borchard F and Joel BS. Carcinoma of the gallbladder: Autopsy findings in 287 cases and review of the literature. J Surg Oncol. 1985;28(3):199-206. https://doi.org/10.1002/jso.2930280311

14. Singh $\mathrm{P}$, Kaur $\mathrm{N}$ and Kaur $\mathrm{M}$. Spectrum of $\mathrm{CT}$ findings in gall bladder carcinoma patients in north Indian population. Int J Med Res Rev. 2017;5(5):499-504 https://doi.org/10.17511/ijmrr.2017.i05.11

15. Yoshimitsu K, Honda H, Shinozaki K, Aibe H, Kuroiwa T, Irie H, et al. Helical CT of the local spread of carcinoma of the gallbladder: Evaluation according to the TNM system in patients who underwent surgical resection. AJR Am J Roentgenol. 2002;179(2):423-428. https://doi.org/10.2214/ajr.179.2.1790423

16. Kalra N, Suri S, Gupta R, Natarajan SK, Khandelwal N, Wig JD, et al. MDCT in the staging of gallbladder carcinoma. AJR Am J Roentgenol. 2006;186(3):758-762. https://doi.org/10.2214/ajr.04.1342

17. Bartlett DL, Fong Y, Fortner JG, Brennan MF and Blumgart LH. Long-term results after resection for gallbladder cancer. Implications for staging and management. Ann Surg. 1996;224(5):639-646. https://doi.org/10.1097V00000658-199611000-00008

18. Ohtani T, Shirai Y, Tsukada K, Muto T and Hatakeyama K. Spread of gallbladder carcinoma: CT evaluation with pathologic correlation. Abdom Imaging. 1996;21(3):195-201. https://doi.org/10.1007/s002619900045

19. Kuszyk BS, Bluemke DA, Urban BA, Choti MA, Hruban RH, Sitzmann JV, et al. Portal-phase contrast-enhanced helical CT for the detection of malignant hepatic tumors: Sensitivity based on comparison with intraoperative and pathologic findings. AJR Am J Roentgenol. 1996;166(1):91-95. https://doi.org/10.2214/ajr.166.1.8571914

20. Coakley FV, Choi PH, Gougoutas CA, Pothuri B, Venkatraman E, Chi D, et al. Peritoneal metastases: Detection with spiral CT in patients with ovarian cancer. Radiology. 2002;223(2):495-499. https://doi.org/10.1148/radiol.2232011081

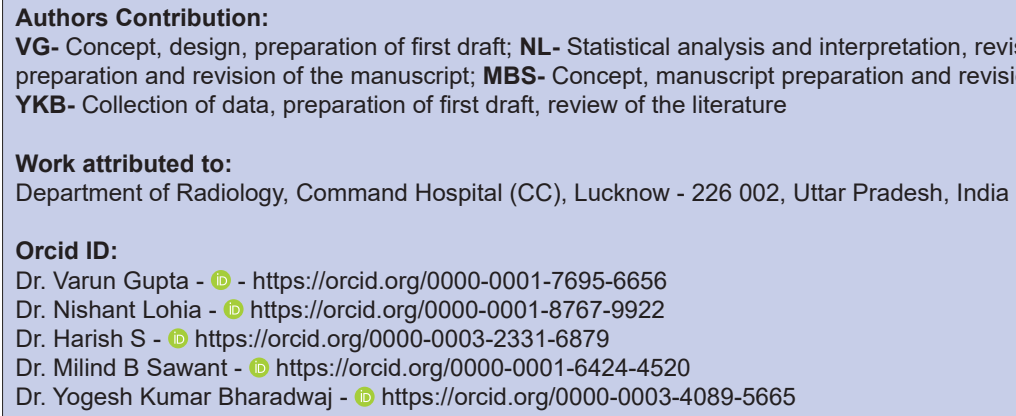

